\title{
Características epidemiológicas de Casos de ofidismo registrados en el Perú durante el período 2010-2019
}

Epidemiological, spatial and temporal distribution of ophidism cases registered in the National Center for Epidemiology, Prevention and Control of Diseases (CDC-PERU) during the period 2010 - 2019

\author{
Gabriela Herrada ${ }^{1}$, Daphne León ${ }^{1}$, Oswaldo Cabanillas ${ }^{2}$
}

\section{RESUMEN}

El objetivo del estudio fue describir la distribución epidemiológica, espacial y temporal de casos de ofidismo registrados en el Centro Nacional de Epidemiología, Prevención y Control de Enfermedades (CDC-PERÚ) en el periodo 2010 - 2019. Para ello se desarrolló un estudio observacional descriptivo, que utilizó la información de casos notificados de ofidismo a nivel nacional, registrados en la Sala Virtual de Situación de Salud, CDC - Perú, para el periodo de estudio. Se obtuvo información de las variables: región, año de ocurrencia, semana epidemiológica expresada en cuartiles e intervalos de cuatro meses, grupo etario y sexo del accidentado. Se diseñó una base de datos con la información obtenida, la que se resumió mediante estadística descriptiva. La asociación entre las variables de agrupación se realizó mediante la prueba de Ji Cuadrado. Se recolectó información de 22564 notificaciones de ofidismo. Los departamentos que registraron la mayor frecuencia de casos durante el periodo en estudio fueron Loreto (28,4\%), San Martín (19,7\%) y Ucayali (13,3\%), que en conjunto sumaron el 61,4\% del total de las notificaciones. Los accidentes en varones $(65,5 \%)$ fueron más frecuentes que en mujeres $(34,5 \%)$. Según estrato etario, los adultos entre 30-59 y 18-29 años sumaron un 64,7 \% del total de casos reportados. En la primera mitad del año, que incorpora el primer (semana 1 a 13) y segundo (semana 14 a 26) cuartil, se presentaron la mayoría de casos (30,7 y $27,4 \%$, respectivamente), que corresponde al $58.8 \%$. La presencia sostenida de casos de ofidismo en el tiempo exige acentuar las medidas de prevención durante todo el año, especialmente en las zonas donde su presencia fue mayor.

PALABRAS CLAVE: anti veneno, serpientes, mordedura, epidemiología.

\section{SUMMARY}

The objective was to describe the epidemiological, spatial and temporal distribution of ophidism cases registered in the National Center for Epidemiology, Prevention and Control of Diseases (CDC-PERU) during the period 2010 - 2019. For this, a descriptive observational study, which used the information of reported cases of ophidism at the national level, registered in the Virtual Health Situation Room, CDC - Peru. Information about region, year of occurrence, epidemiological week expressed in quartiles and four-month intervals, age group and sex of the injured person, was obtained. The information obtained was summarized using descriptive statistics and the association between variables was made using the Chi Square test. Information was collected from 22,564 reports of ophidism. The departments that registered the highest frequency of cases during the study period were Loreto (28.4\%), San Martín (19.7\%) and Ucayali (13.3\%), which together accounted for $61.4 \%$ of the total notifications. Accidents in men $(65.5 \%)$ were more frequent than in women (34.5\%). According to age group, adults between 30-59 and 18-29 years

Laboratorio de Epidemiología y Salud Pública en Veterinaria, Facultad de Medicina Veterinaria y Zootecnia, Universidad Peruana Cayetano Heredia.Lima, Perú 
old accounted for $64.7 \%$ of the total reported cases. In the first half of the year, which incorporates the first (week 1 to 13 ) and second (week 14 to 26) quartile, the majority of cases occurred (30.7 and 27.4\%, respectively), which corresponds to $58.8 \%$. The sustained presence of cases of ophidism over time requires accentuating prevention measures throughout the year, especially in areas where its presence was greater.

KEYWORDS: anti venom, snakes, bite, epidemiology.

\section{INTRODUCCIÓN}

Los accidentes ofídicos corresponden a una lesión cutánea seguida de la inoculación de la sustancia tóxica o veneno, el cual produce daño a los tejidos y ocasiona alteraciones fisiopatológicas a nivel local o sistémico de gravedad variable (Juárez-Villa et al., 2016). El ofidismo es la principal causa de envenenamientos fatales causados por animales ponzoñosos en el Perú (Zavaleta, 2004) y está considerado dentro del grupo de enfermedades infecciosas desatendidas según clasificación y priorización de la OMS, por lo que se demanda su atención (Cabezas-Sánchez, 2014).

El género Bothrops es considerado el principal responsable de los accidentes ofídicos en el Perú y Latinoamérica (Vera et al., 2006; Boadas et al., 2012). Las manifestaciones clínicas varían de acuerdo a su gravedad. La manifestación local suele presentarse con mayor frecuencia dolor, edema y eritema. Las manifestaciones sistémicas expresan fiebre, cefalea y mareo/nauseas. Las manifestaciones hemorrágicas muestran anemia, gingivorragia, equimosis, leucocitosis y tiempo de coagulación prolongado (Lévano y Fernández, 2004).

La atención de los accidentes ofídicos es un requerimiento urgente. La administración del suero antiofídico es el principal tratamiento, el cual debe de ser suministrado tempranamente y en cantidad terapéutica (Vera 2016). El uso de corticoides, antibióticos y fluidoterapia, complementan el tratamiento del accidente ofídico en las personas (Segura et al., 2013; Pezo, 2017).

Los accidentes ofídicos están asociados principalmente a la residencia en zona rural y actividades agrícolas, lo que afecta especialmente a adultos jóvenes de género masculino durante sus labores en el campo (Vera et al., 2006; Boadas et al., 2012; Gordo et al., 2016); por ello, es necesario educar a la población en riesgo, sobre las medidas de prevención ante este tipo de accidentes (Vera et al., 2006; Riofrio et al., 2018).
Dado que el ofidismo es considerado como un problema de importancia en la salud pública, el objetivo del estudio fue describir la distribución epidemiológica, espacial y temporal de casos de ofidismo registrados en la Sala Virtual de Situación de Salud, CDC - Perú en el periodo 2010 - 2019.

\section{MATERIAL Y MÉTODOS}

El estudio se realizó en el Laboratorio de Epidemiología y Salud Pública en Veterinaria de la Facultad de Medicina Veterinaria y Zootecnia de la Universidad Peruana Cayetano Heredia. La investigación correspondió a un estudio observacional descriptivo.

La población de estudio correspondió a la totalidad de los casos de ofidismo notificados en el CDCPERÚ en el periodo 2010 - 2019, base que recoge la información reportada por las Direcciones de Redes Integradas de Salud (DIRIS, antes Direcciones de Salud - DISA) y las Direcciones y Gerencias Regionales de Salud (DIRESAS/GERESAS). Esta información se encuentra a disposición del público en el portal de la Sala Virtual de Situación de Salud, CDC - Perú.

El estudio recuperó información de las siguientes variables:

- Departamento que reportó los accidentes según registró del Centro Nacional de Epidemiología, Prevención y Control de Enfermedades (CDCPerú). Periodo 2010-2019.

- Año de ocurrencia (el periodo de estudio evaluado fue 2010-2019)

- Grupo etario ( $<1$ año, 1 a 4 años, 5 a 11 años, 12 a 14 años, 15 a 17 años, 18 a 29 años, adulto 30 a 59 años, adultos mayores de 60 a más años).

- Sexo (masculino y femenino).

- Semana epidemiológica expresada en cuartiles (Semana 1 a 13, 14 a 26, 27 a 39 y 40 a 52/53) y a intervalos de 4 semanas (aproximación a meses)

El análisis estadístico se realizó en STATA 15.0. Los resultados se resumieron mediante estadística 
descriptiva (frecuencias absolutas y relativas) y se presentan a través de tablas. La asociación entre variables se determinó mediante la prueba de Ji cuadrado. El estudio fue aprobado por el Comité Institucional de Ética de la Universidad Peruana Cayetano Heredia mediante constancia E035-10-20.

\section{RESULTADOS}

El estudio recolectó información de 22579 notificaciones de ofidismo registrados en el portal de la Sala Virtual de Situación de Salud, CDC - Perú, en el periodo del 2010 al 2019. Los departamentos que registraron la mayor cantidad de casos durante el periodo en estudio fueron Loreto (28,4\%), San Martín $(19,7 \%)$ y Ucayali $(13,3 \%)$, que en conjunto sumaron el $61,4 \%$ del total de las notificaciones (tabla 1 ).

En cuanto el sexo de las personas afectadas, predominaron los casos en varones $(65,5 \%)$ sobre los reportados en mujeres $(34,5 \%)$, las proporciones fueron significativamente diferentes $(\mathrm{p}<0,01)$ (tabla 2). Por otro lado, la distribución de los casos según estrato etario muestra que los adultos entre 18-29 y 30-59 años sumaron un $64,7 \%$ del total de casos reportados en el periodo de estudio (25,1 y $39,6 \%$, respectivamente). Las proporciones entre los grupos etarios fueron significativamente diferentes $(\mathrm{p}<0,01)$

Tabla 1. Distribución por departamento de los casos de ofidismo registrados en el Centro Nacional de Epidemiología, Prevención y Control de Enfermedades (CDC-Perú). Periodo 2010-2019.

\begin{tabular}{|c|c|c|}
\hline \multirow{2}{*}{ Departamento } & \multicolumn{2}{|c|}{ Casos reportados } \\
\hline & Nro. & $\%$ \\
\hline Loreto & 6419 & 28,4 \\
\hline San Martin & 4451 & 19,7 \\
\hline Ucayali & 3009 & 13,3 \\
\hline Amazonas & 1982 & 8,8 \\
\hline Junín & 1536 & 6,8 \\
\hline Huánuco & 1318 & 5,8 \\
\hline Cuzco & 875 & 3,9 \\
\hline Pasco & 658 & 2,9 \\
\hline Madre de Dios & 623 & 2,8 \\
\hline Piura & 483 & 2,1 \\
\hline Cajamarca & 250 & 1,1 \\
\hline Lima & 247 & 1,1 \\
\hline La Libertad & 217 & 1,0 \\
\hline Ayacucho & 190 & 0,8 \\
\hline Puno & 104 & 0,5 \\
\hline Lambayeque & 87 & 0,4 \\
\hline Tumbes & 58 & 0,3 \\
\hline Huancavelica & 29 & 0,1 \\
\hline Ancash & 28 & 0,1 \\
\hline Ica & 9 & $<0,01$ \\
\hline Arequipa & 3 & $<0,02$ \\
\hline Callao & 3 & $<0,03$ \\
\hline Apurímac & 0 & 0 \\
\hline TOTAL & 22579 & 100 \\
\hline
\end{tabular}


Tabla 2. Distribución, según año y sexo, de los casos de ofidismo registrados en el Centro Nacional de Epidemiología, Prevención y Control de Enfermedades (CDC-Perú). Periodo 2010-2019.

\begin{tabular}{ccccccc}
\hline \multirow{2}{*}{ Año } & \multirow{2}{*}{ Total de casos } & \multicolumn{2}{c}{ Femenino } & & \multicolumn{2}{c}{ Masculino } \\
\cline { 3 - 4 } \cline { 6 - 7 } & & $\mathbf{n}$ & $\mathbf{\%}$ & & $\mathbf{n}$ & $\mathbf{\%}$ \\
\hline 2010 & 2425 & 810 & 33,4 & & 1615 & 66,6 \\
2011 & 2497 & 897 & 35,9 & & 1600 & 64,1 \\
2012 & 2375 & 837 & 35,2 & & 1538 & 64,8 \\
2013 & 2281 & 773 & 33,9 & & 1508 & 66,1 \\
2014 & 2144 & 744 & 34,7 & & 1400 & 65,3 \\
2015 & 2212 & 779 & 35,2 & & 1433 & 64,8 \\
2016 & 2254 & 798 & 35,4 & & 1456 & 64,6 \\
2017 & 2119 & 718 & 33,9 & & 1401 & 66,1 \\
2018 & 2185 & 741 & 33,9 & & 1444 & 66,1 \\
2019 & 2087 & 689 & 33 & & 1398 & 67 \\
Total & 22579 & 7786 & 34,5 & & 14793 & 65,5 \\
\hline
\end{tabular}

Tabla 3. Accidentes ofídicos, distribuidos por año y grupo etario, registrados en el Centro Nacional de Epidemiología, Prevención y Control de Enfermedades (CDC-Perú). Periodo 2010-2019.

\begin{tabular}{|c|c|c|c|c|c|c|c|c|c|c|c|c|c|c|c|c|c|}
\hline \multirow[t]{2}{*}{ Año } & \multirow{2}{*}{$\begin{array}{l}\text { Total } \\
\text { de } \\
\text { casos }\end{array}$} & \multicolumn{2}{|c|}{$\begin{array}{l}<1 \\
\text { año }\end{array}$} & \multicolumn{2}{|c|}{$\begin{array}{c}1-4 \\
\text { Años }\end{array}$} & \multicolumn{2}{|c|}{$\begin{array}{l}5-11 \\
\text { años }\end{array}$} & \multicolumn{2}{|c|}{$\begin{array}{l}12-14 \\
\text { años }\end{array}$} & \multicolumn{2}{|c|}{$\begin{array}{l}\text { 15-17 } \\
\text { años }\end{array}$} & \multicolumn{2}{|c|}{$\begin{array}{l}\text { 18-29 } \\
\text { años }\end{array}$} & \multicolumn{2}{|c|}{$\begin{array}{c}30-59 \\
\text { años }\end{array}$} & \multicolumn{2}{|c|}{$\begin{array}{c}60 \mathrm{a}+ \\
\text { años }\end{array}$} \\
\hline & & $\mathrm{n}$ & $\%$ & $\mathrm{n}$ & $\%$ & $\mathrm{n}$ & $\%$ & $\mathrm{n}$ & $\%$ & n. & $\%$ & $\mathbf{n}$ & $\%$ & $\mathbf{n}$ & $\%$ & $\mathrm{n}$ & $\%$ \\
\hline 2010 & 2425 & 3 & 0,1 & 75 & 3,1 & 287 & 11,8 & 169 & 7,0 & 198 & 8,2 & 670 & 27,6 & 860 & 35,5 & 163 & 6,7 \\
\hline 2011 & 2497 & 5 & 0,2 & 86 & 3,4 & 284 & 11,4 & 165 & 6,6 & 196 & 7,8 & 675 & 27,0 & 924 & 37,0 & 162 & 6,5 \\
\hline 2012 & 2375 & 4 & 0,2 & 63 & 2,7 & 261 & 11,0 & 156 & 6,6 & 181 & 7,6 & 630 & 26,5 & 898 & 37,8 & 182 & 7,7 \\
\hline 2013 & 2281 & 4 & 0,2 & 59 & 2,6 & 243 & 10,7 & 166 & 7,3 & 173 & 7,6 & 576 & 25,3 & 885 & 38,8 & 175 & 7,7 \\
\hline 2014 & 2144 & 2 & 0,1 & 69 & 3,2 & 218 & 10,2 & 149 & 6,9 & 156 & 7,3 & 567 & 26,4 & 815 & 38 & 168 & 7,8 \\
\hline 2015 & 2212 & 6 & 0,3 & 47 & 2,1 & 239 & 10,8 & 141 & 6,4 & 144 & 6,5 & 567 & 25,6 & 895 & 40,5 & 173 & 7,8 \\
\hline 2016 & 2254 & 2 & 0,1 & 46 & 2,0 & 237 & 10,5 & 142 & 6,3 & 149 & 6,6 & 543 & 24,1 & 944 & 41,9 & 191 & 8,5 \\
\hline 2017 & 2119 & 2 & 0,1 & 52 & 2,5 & 209 & 9,9 & 145 & 6,8 & 127 & 6,0 & 490 & 23,1 & 909 & 42,9 & 185 & 8,7 \\
\hline 2018 & 2185 & 1 & 0,0 & 45 & 2,1 & 229 & 10,5 & 127 & 5,8 & 159 & 7,3 & 477 & 21,8 & 941 & 43,1 & 206 & 9,4 \\
\hline 2019 & 2087 & 1 & 0,0 & 45 & 2,2 & 232 & 11,1 & 142 & 6,8 & 129 & 6,2 & 465 & 22,3 & 868 & 41,6 & 205 & 9,8 \\
\hline Total & 22579 & 30 & 0,1 & 587 & 2,6 & 2439 & 10,8 & 1501 & 6,7 & 1611 & 7,1 & 5655 & 25,1 & 8931 & 39,6 & 1810 & 8,0 \\
\hline
\end{tabular}

entre las tres proporciones mayores entre sí y estos respecto a los demás (tabla 3 ).

La distribución de los casos según cuartiles muestra que el primer cuartil (semana 1 a 13) y segundo cuartil (semana 14 a 26) del año, presentaron la mayor proporción de casos durante el periodo de estudio (30,7 y $27,4 \%$, respectivamente). Las proporciones entre los cuartiles fueron significativamente diferentes $(p<0,01)$ entre todos (tabla 4).

\section{DISCUSIÓN}

Los resultados del estudio muestran que el ofidismo en el Perú es un problema importante de salud pública cuyo impacto se puede agravar cuando se ve limitado el acceso a un tratamiento adecuado y oportuno. Pesantes-Segura (2000) menciona que el ofidismo ha sido causa de pérdidas de vidas humanas y cuando ello no sucede, las personas sufren de destrucción masiva de tejidos lo que produce la pérdida total o parcial de 
Tabla 4. Accidentes ofídicos, distribuidos por año y cuartiles, registrados en el Centro Nacional de Epidemiología, Prevención y Control de Enfermedades (CDC-Perú). Periodo 2010-2019.

\begin{tabular}{cccccccccc}
\hline \multirow{2}{*}{ Año } & \multirow{2}{*}{ TOTAL } & \multicolumn{2}{c}{ Semana 1-13 } & \multicolumn{2}{c}{ Semana 14-26 } & \multicolumn{2}{c}{ Semana 27-39 } & \multicolumn{2}{c}{ Semana 40-52/53 } \\
\cline { 3 - 9 } & & $\mathbf{n}$ & $\mathbf{\%}$ & $\mathbf{n}$ & $\mathbf{\%}$ & $\mathbf{n}$ & $\mathbf{\%}$ & $\mathbf{n}$ & $\mathbf{\%}$ \\
\hline 2010 & 2425 & 793 & 32,7 & 658 & 27,1 & 498 & 20,5 & 476 & 19,6 \\
2011 & 2497 & 710 & 28,4 & 751 & 30,1 & 503 & 20,1 & 533 & 21,3 \\
2012 & 2375 & 720 & 30,3 & 619 & 26,1 & 500 & 21,1 & 536 & 22,6 \\
2013 & 2281 & 729 & 32 & 635 & 27,8 & 429 & 18,8 & 488 & 21,4 \\
2014 & 2144 & 593 & 27,7 & 639 & 29,8 & 427 & 19,9 & 485 & 22,6 \\
2015 & 2212 & 670 & 30,3 & 569 & 25,7 & 484 & 21,9 & 489 & 22,1 \\
2016 & 2254 & 776 & 34,4 & 541 & 24 & 496 & 22 & 441 & 19,6 \\
2017 & 2119 & 649 & 30,6 & 585 & 27,6 & 451 & 21,3 & 434 & 20,5 \\
2018 & 2185 & 635 & 29,1 & 640 & 29,3 & 437 & 20 & 473 & 21,6 \\
2019 & 2087 & 647 & 31 & 538 & 25,8 & 380 & 18,2 & 522 & 25,0 \\
TOTAL & 22579 & 6920 & 30,7 & 6174 & 27,4 & 4604 & 20,4 & 4866 & 21,6 \\
\hline
\end{tabular}

algún miembro, incapacitándolas permanentemente para el desarrollo de sus actividades rutinarias.

Los departamentos con mayor número de casos durante el periodo en estudio fueron Loreto, San Martín y Ucayali. En estos lugares la distancia entre la urbanización y la zona rurales se ha ido estrechando, tanto físicamente como por el desarrollo de actividades económicas que la población realiza para asegurar la alimentación de la familia; esto favorece el ofidismo. En ese sentido, Villanueva et al. (2004) mencionan que la introducción del hombre hacia zonas rurales y la colonización de la amazonia con propósitos extractivos o agrícolas, favorecería a una mayor exposición de las personas a los accidentes ofídicos; y pueden ser de desenlace fatal cuando ocurren en zonas alejadas, situadas a muchas horas de un centro de atención médica.

En la misma línea, Vera (2016) y García-Willis et al. (2009) mencionan que los accidentes ofídicos se presentan con mayor frecuencia en zonas rurales y que la población vulnerable incluye a los agricultores debido al inadecuado o nulo uso de medidas de protección en el área laboral; por lo que Riofrio et al. (2018) recomiendan educar a la población en la identificación de los factores de riesgo y las medidas de prevención de estos accidentes.

Una actividad económica importante en la región es el turismo de aventura. En ese sentido, Manrique (2000) considera que el turismo vivencial o de aventura es un factor que expone al ofidismo. Las zonas tropicales de Perú suelen ser destino para este tipo de actividades, de allí que los departamentos como Loreto, San Martin y Ucayali, que son explotados para el turismo (Carrillo et al., 2017), presentan también una gran cantidad de accidentes por ofidios. Una situación contraria se encuentra en los departamentos de la costa peruana, en donde los accidentes por mordedura de serpiente tienen frecuencias menores. Rodríguez y Calderón (1991) mencionaron que con el aumento de las zonas urbanas se habría eliminado prácticamente el hábitat de las serpientes venenosas que antes eran abundantes.

En los accidentes ofídicos reportados durante el periodo de estudio se encontró que los más afectados fueron los varones y los grupos etarios adultos, quienes suelen estar expuestos debido a su actividad laboral. Similares resultados encuentran Vera et al. (2006) en Paraguay, Gordo et al. (2016) en Magdalena Colombia, Morejón et al. (2006) y De Andrade (2015) en Brasil.

En el Perú, Villanueva et al. (2004), encuentra que en la provincia de Chanchamayo, Junín, la edad media de los afectados con accidentes ofídicos fue de 26.2 años y que la mayoría de los afectados eran de sexo masculino (62.4\%). Más adelante, Bernabé (2011) encontró que el $50 \%$ de los afectados en la ciudad de Satipo correspondía a personas de 11 a 30 años de edad y coincide que el sexo más afectado era el masculino $(60 \%)$. En estos dos últimos estudios las edades de los afectados abarcaron un rango más amplio que el reportado en este estudio. 
Los niños también pueden representar una población vulnerable en zonas endémicas a ofidios y las mordeduras se pueden presentar en lugares donde ellos desarrollan sus actividades diarias. González (2017) encontró que los accidentes ocurrieron principalmente en zona escolar $(60 \%)$, seguido del entorno de la vivienda o dentro de la misma (21\%) y en menor proporción en el campo como zona de distracción o lugar donde trabajaban algunos de ellos (19\%).

El estudio encuentra también que un mayor número de casos de ofidismo se presentó en la primera mitad del año, que incluía parte de las estaciones de verano y otoño, épocas donde la temperatura ambiental es la mayor. Similar tendencia se encuentra en los resultados de Vera et al. (2006) en Paraguay, quienes observaron una mayor frecuencia de accidentes ofídicos entre los meses de marzo y abril (30\%). Así mismo, De Andrade (2015) en Brasil encontró que aproximadamente el 51\% de los accidentes ocurrió en el verano. También se encuentra un patrón similar en Perú, donde Villanueva et al. (2004) encontraron que los casos de ofidismo ocurrieron con mayor frecuencia (67\%) durante los meses de lluvia (diciembre a mayo).

Estudios realizados en la década de los $80 \mathrm{~s}$ como los de Pernaz (1982), Valladolid et al. (1983), Maguiña y Lumbreras (1986), Chang y Zavaleta (1987) y Zavaleta (2004), reportan entre 55,4 y 69\% de accidentes ofídicos se produjeron durante los meses lluviosos (noviembre a marzo). Esta situación se explicaba, según los autores, por la necesidad de las especies ponzoñosas de trasladarse cerca de las viviendas humanas, toda vez que el crecimiento de los ríos las obliga a subir las riberas, lo que aumenta la posibilidad de un encuentro casual con el hombre.

Aunque la base de datos de CDC - Perú no ofrece información del tipo de serpiente que se encuentra involucrada en el ofidismo, una serie de investigaciones sindican al género Bothrops sp., encontrarse involucrados en la mayoría de ellos. Yarlequé (2000) identifico cuatro tipos de accidentes ofídicos: los Bothrópico, Crotálico, Lachésico y Elapídico; resaltó que el primero fue el más frecuente, este alcanzó tasas del 70\%. En Paraguay Vera et al. (2006) encontraron que el género Bothrops sp. fue el responsable del 92\% de los accidentes y en Brasil, Morejón et al. (2006) encuentra que esta proporción llegó al 63,3\%.

Para enfrentar de manera eficiente un accidente ofídico se hace necesario conocer el género de serpiente mordedora para así reconocer el tipo de efecto tóxico que producirá veneno, por ello conocer la distribución geográfica de las serpientes también es necesario. Yarlequé (1992) describe el modo de acción del veneno de los ofidios venenosos más comunes en Perú, el veneno del género Bothrops es proteolítico (coagulante-hemorrágico), el cual se manifiesta con intenso dolor y edema que se extiende a todo el miembro afectado. A ello se agrega precozmente flogosis, equimosis y/o flictenas hemorrágicas en el sitio de la mordedura. A nivel sistémico se presentan cuadros hemorrágicos locales, coagulación intravascular diseminada y trombosis. El veneno del género Crotalus es de tipo mionecrótico y neurotóxico; el del género Lachesis es de tipo proteolítico, coagulante e hipotensor y el veneno del género Micrurus es principalmente neurotóxico.

La localización anatómica de la mordedura suele estar asociada a la actividad que realizaba la persona al momento de ocurrir el accidente. Maguiña et al. (1998) y Villanueva et al. (2004) mencionaron que el miembro inferior fue la localización más común en regiones selváticas, debido a que ellos se producen durante el desarrollo del trabajo agrícola o durante el desplazamiento en caminos rurales. En contraste, la localización anatómica más afectada en la costa suele ser el miembro superior, ya que se producen durante actividades de recreación o en accidentes laborales (personal que labora en serpentarios).

El tratamiento principal para enfrentar el accidente ofídico es la administración del suero antiofídico (antídoto). Zavaleta (2004) mencionó que el suero antiofídico es un producto escaso a nivel mundial. En Perú la producción es realizada por el Instituto Nacional de Salud cuya producción es pequeña e insuficiente para cubrir la demanda nacional, por lo que se requiere su importación (Centro Nacional de Abastecimiento de Recursos Estratégicos en Salud [CENARES], 2020). Ello demanda desarrollar actividades de promoción de la salud a fin de evitar la exposición a los accidentes ofídicos en la población que habita zonas endémicas de serpientes ponzoñosas.

Se sabe que los accidentes ofídicos no se encuentran priorizados por los sistemas de salud debido a que la mayoría de ellos son producidos en área rural, por lo tanto, es limitado su registro, así como su atención con los recursos médicos adecuados. Quesada y Quesada (2012) mencionaron que los afectados suelen llegar a los servicios de salud después de haber recibido un tratamiento no convencional. A ello se suma lo 
mencionado por Izaguirre-González et al. (2015) quienes señalan que las mordeduras de serpiente son motivos de consulta poco habitual en los servicios de urgencias, por lo que pueden encontrarse dudas sobre el tratamiento a aplicar, particularmente al decidir sobre el uso de un antídoto específico. Por ello es necesario que en zonas endémicas el estado abastezca a los servicios de salud de suero antiofídico. Tomando como base la información histórica de la presentación de casos, la misma que forma parte de sus bases de datos de donde este estudio ha obtenido la información.

\section{CONCLUSIONES}

El estudio "Características epidemiológicas de casos de ofidismo registrados en el portal del Centro Nacional de Epidemiología, Prevención y Control Enfermedades (CDC-PERÚ) durante el período 20102019" llega a las siguientes conclusiones:

- El ofidismo es un problema de salud pública que afecta principalmente a la población que reside en los departamentos de Loreto, San Martín y Ucayali por lo que se requiere priorizar medidas preventivas en estas zonas.

- La mayor cantidad de casos de ofidismo se presentaron en personas del sexo masculino y de edades productivas por lo que además del impacto sobre la salud de las personas, este problema puede afectar la economía de los hogares que dependen de los afectados.

- El ofidismo se presenta en forma endémica durante el periodo 2009-2019 por lo que se debe de reevaluar las medidas de prevención en busca de disminuir su incidencia.

\section{Correspondencia}

Daphne León Córdova

Correo electrónico: daphne.leon@upch.pe

\section{REFERENCIAS BIBLIOGRAFICAS}

1. Bernabé, D. (2011). Características clínicas del ofidismo en el Hospital Manuel Ángel Higa ArakakiSatipo 2009-2010. [Tesis para Título Profesional de Medico Citujano, Universidad Nacional del Centro]. Repositorio Institucional Digital. http://repositorio. uncp.edu.pe/bitstream/handle/UNCP/617/TMH_103. pdf?sequence $=1$

2. Boadas, J., Matos, M., Bónoli, S., Borges, A., Vásquez-Suárez, A., Serrano, L. \& De Sousa, L. (2012). Perfil eco-epidemiológico de los accidentes por ofidios en Monagas, Venezuela (2002-
2006). Boletín de malariología y salud ambiental, 52(1), 107-120.

3. Cabezas-Sánchez, C. (2014). Enfermedades infecciosas desatendidas: un permanente reto para la salud pública y la equidad en el Perú. Revista Peruana de Medicina Experimental y Salud Pública, 31, 326335.

4. Centro Nacional de Abastecimiento de Recursos EstratégicosenSalud.(2020).Adquisicióndeproductos de Zoonosis. Suero Antiofidico Polivalente Liofilizado. Centro Nacional de Abastecimiento de Recursos Estratégicos en Salud. http:// www.cenares.minsa.gob.pe/Portals/0/Descargas/ ComprasInternacionales/2020/ETI\%20SUERO\%20 ANTIOFIDICO.pdf

5. Carrillo, O., Dongo, P., Guerrero de la Cruz, P., \& Ortiz, F. (2017). Planeamiento estratégico para la Provincia de San Martín. [Tesis Magister]. Pontifica Universidad Católica del Perú.

6. Chang, J., \& Zavaleta, A. (1987). Ofidismo en el Hospital General de La Merced: Estudio retrospectivo de 116 casos. Diagnóstico, 20(4), 115-120.

7. De Andrade, A. (2015). Análise clínicoepidemiológica de casos de ofidismo atendidos em um hospital público estadual de Minas Gerais de 2003 a 2012. [Tesis de Maestría]. Universidade Federal de Minas Gerais.

8. García-Willis, C.E., Vela-Ortega, R., \& Maya-Leal, M. E. (2009). Epidemiología de la mordedura por ofidio en pacientes pediátricos. Boletín médico del Hospital Infantil de México, 66(3), 254-259.

9. González, P. F. (2017). Mordeduras de serpientes en pacientes pediátricos de 1 a 14 años atendidos en el Hospital Francisco Icaza Bustamante en el periodo de 2016. [Tesis Título]. Universidad de Guayaquil.

10. Gordo, L.C.C., Orozco, B. A., Goenaga, G. O., Ortiz, Y. M. B., \& Otálvaro, J. P. (2016). Comportamiento epidemiológico del accidente ofídico en el Departamento del Magdalena, Colombia (20092013). Revista Ciencias de la Salud, 14(2), 161-177.

11. Izaguirre-González, A., Matute-Martínez, C. F., Barahona-López, D. M., Sánchez-Sierra, L. E., \& Perdomo-Vaquero, R. (2015). Caracterización clínico-epidemiológica de mordedura de serpiente en el Hospital Regional Santa Teresa de Comayagua, 2014-2015. Rev Med Hondur, 85(1-2), 21-26.

12. Juárez-Villa, J. D., López-De León, J., RodríguezMandujano, M., \& Echegollen-Guzmán, A. (2016). Mordedura por serpiente en el Hospital General de Ciudad Victoria, Tamaulipas. Evid Med Invest Salud, 9, 92-7.

13. Lévano, J., \& Fernández, R. (2004). Diagnóstico y tratamiento de los accidentes por animales ponzoñosos.Instituo Nacional de Salud. https:// repositorio.ins.gob.pe/bitstream/handle/INS/118/ CNPB-0003.pdf? sequence $=3 \&$ is Allowed $=y$ 
14. Maguiña, C., Henríquez, C., Ilquimiche, L., Mostorino, R., Gotuzzo, E., \& Legua, P. (1998). Ofidismo por Bothrops pictus en el Hospital Nacional Cayetano Heredia: Estudio prospectivo de 23 casos. Folia Dermatol Perú, 9, 1-2. http://sisbib. unmsm.edu.pe/bvrevistas/folia/vol9_n1-2/ofidismo. htm

15. Maguiña, C., \& Lumbreras, H. (1986). Ofidismo en el Hospital Cayetano Heredia (1976-1986). [Resumen]. IV Jornada Universidad Peruana Cayetano Heredia. Lima; 8-19 Set 1986.

16. Manrique, C.H.A. (2000). Ofidismo. Módulos Técnicos. Serie Documentos Monográficos. Instituto Nacional de Salud - Ministerio de Salud.

17. Morejón, M., Díaz, S., \& Rosa, R. (2006). Ofidismo: Estudio de 30 casos en Brasil. Revista Cubana de Medicina General Integral, 22(2), 0-0. http://scielo. sld.cu/pdf/mgi/v22n2/mgi18206.pdf

18. Pernaz, G. (1982). Ofidismo: estudio retrospectivo de 103 casos en el Hospital General de La Merced (Chanchamayo - Junín). [Tesis de Bachiller en Medicina]. Universidad Peruana Cayetano Heredia.

19. Pesantes-Segura, O. (2000). Prevención y tratamiento de accidentes por serpientes venenosas. SEDAPAL.

20. Pezo, J. (2017). Características epidemiológicas, clínica y tratamiento de pacientes atendidos por accidente ofídico bothrópico. Hospital II-2 TarapotoMINSA. 2013-2015. [Tesis Bachiller) Universidad Nacional de San Martín-Tarapoto.

21. Quesada, J. A., \& Quesada, E. Q. (2012). Prevención y manejo de mordeduras de serpientes. Revista Archivo Médico de Camagüey, 16(3), 369-383.

22. Riofrio-Pinargote, C. A., Duran-Pincay, Y. E., PincayParrales, E. G., Duran-Ávila, N. L., Baque-Pibaque, A. A., \& Loor-Choez, E. N. (2018). Aspectos clínicos y epidemiológicos de los accidentes ofídicos del cantón Jipijapa. Polo del Conocimiento, 3(7), 664676 .
23. Rodríguez, J., \& Calderón, J. (1991). Ofidismo en el Hospital de Apoyo de Tarapoto 1968-1990. Acta Médica, 15(2), 9-15.

24. Segura, M., Hernández, H., Falcón, N., \& Silva, W. (2013). Accidentes por animales ponzoñosos en pacientes internados en un hospital de niños en Lima, Perú. Estudio retrospectivo en el periodo 20002009. Salud tecnol vet, 1, 52-59.

25. Valladolid, J., Cruz, J., \& Angeles, A. (1983). Mordeduras de Serpientes: Hospital Yurimaguas, 1974-1981. [Resúmenes de Temas Libres]. III Congreso Regional de Medicina. Trujillo, Perú.

26. Vera, A. (2016). Prevalencia; caracterización clínica y terapéutica de los accidentes ofídicos en el Hospital Napoleón Dávila de Chone. Manabí. Periodo 2005-2011. Propuesta de protocolo de atención [Tesis Maestría]. Universidad de Guayaquil.

27. Vera, A., Páez, M., \& de Cáceres, G. G. (2006). Caracterización Epidemiológica de los accidentes ofídicos, Paraguay 2004. Memorias del Instituto de Investigaciones en Ciencias de la Salud, 4(1),0-0. http://revistascientificas.una.py/index.php/RIIC/ article/view/351/277

28. Villanueva, M., Maguiña, C., Cabada, M. M., De Marini, J., Alvarez, H., \& Gotuzzo, E. (2004). Ofidismo en la provincia de Chanchamayo, Junín: revisión de 170 casos consecutivos en el Hospital de Apoyo de La Merced. Revista Medica Herediana, 15(2), 82-87. http://www.scielo.org.pe/ $\mathrm{pdf} / \mathrm{rmh} / \mathrm{v} 15 \mathrm{n} 2 / \mathrm{v} 15 \mathrm{n} 2 \mathrm{ao} 4 . \mathrm{pdf}$

29. Yarlequé A. (1992). Investigación en venenos de serpientes. Alma Máter UNMSM, 2, 61-77.

30. Yarlequé, L. (2000). Las serpientes peruanas y sus venenos. Fondo Editorial de la Universidad Nacional Mayor de San Marcos.

31. Zavaleta, A. (2004). Mordedura de serpiente (ofidismo): Un problema de salud en el Perú. Rev Méd Hered, 15(2), 61-63. 